\title{
RITMOS CIRCADIANOS DE ACTIVIDAD LOCOMOTORA EN RHAMDIA QUELEN ${ }^{1}$
}

\author{
Scaglione, M. C. ${ }^{2}$; Gervasoni, S. h. ${ }^{2}$; Garbe, n. e. ${ }^{2}$; \\ Marozzi, M. ${ }^{2}$ \& Cerutti, R.D. ${ }^{2}$
}

\begin{abstract}
RESUMEN
En este trabajo se analizan los ritmos de actividad locomotora del Rhamdia quelen (Quoy \& Gaimard, 1824), pez teleósteo de interés en acuicultura en Sudamérica. Se llevaron a cabo dos experimentos con 30 animales, mantenidos por tríos en estanques de 100 litros. Cada acuario tenía un sensor infrarrojo para registrar el movimiento de los peces. En el primer experimento se caracterizó la ritmicidad diaria de actividad reposo bajo condiciones de luz-oscuridad LO 12:12. El segundo experimento fue diseñado para estudiar el marcapaso endógeno. Para ello se invirtió el ciclo LO para analizar la resincronización. Posteriormente los ritmos circadianos se estudiaron bajo condiciones de curso libre en luz constante y pulsos ultradianos. Nuestros resultados muestran que $R$. quelen es una especie estrictamente diurna. Los ritmos de actividad diaria que se resincronizan rápidamente tras invertir el ciclo LO y persisten bajo condiciones de curso libre LL y pulsos cortos, sugiriendo un origen circadiano.

Palabras claves: cronobiología, ritmos biológicos, fotoperiodo, actividad locomotora, Rhamdia quelen.
\end{abstract}

\section{SUMMARY}

\section{Circadian rhythms of the locomotor activity in Rhamdia quelen (South American catfish).}

In this paper were analyzed the rhythms of locomotor activity in Rhamdia quelen (Quoy \& Gaimard, 1824), teleost fish of interest in South American aquaculture. For this, two experiments were performed. Ten groups of 3 individuals each were maintained in tanks of 100 liters. In each aquarium, it was used infrared sensors in order to record the movement of fish. In the first experiment the daily rhythmicity of activity-rest was characterized under 12:12 hours light-dark (LD) conditions. The

1.- Subsidiado por SECTeI de la Provincia de Santa Fe como Apoyo a proyectos de investigación sobre temas de interés provincial desarrollados por Grupos de Investigación pertenecientes al sistema de Ciencia, Tecnología e Innovación, con Institución Adoptante.

2.- Facultad de Ciencias Veterinarias, Universidad Nacional del Litoral. Kreder 2805. Esperanza, provincia de Santa Fe. Email: mcscagli@fcv.unl.edu.ar

Manuscrito recibido el 27 de noviembre de 2013 y aceptado para su publicación el $1^{\circ}$ de abril de 2014 . 
second experiment was designed to study the endogenous pacemaker. In that sense, the LO cycle was reversed to analyze resynchronization. Subsequently the circadian rhythms were studied under free course in constant light and ultradian pulses. Our results showed that the R. quelen is a strictly diurnal species, the rhythms of daily activity resynchronized quickly after investing the LO cycle and persist under free course LL and short pulses, suggesting a circadian origin.

Key words: chronobiology, biological rhythms, photoperiod, locomotor activity, Rhamdia quelen

\section{INTRODUCCIÓN}

Los ritmos biológicos no constituyen un fenómeno casual ni resultan de un seguimiento pasivo de las condiciones ambientales, sino que forman parte del proceso de adaptación de los seres vivos al ambiente. $\mathrm{Su}$ importancia radica en que ajustan los procesos internos con los cambios periódicos externos, preparando así al organismo para situaciones predecibles y repetitivas (Aschoff, 1981). Estos ritmos son de origen genético (Hardin et al., 1990), y son característicos de cada especie animal, aunque pueden observarse variaciones interindividuales. Se sabe que el patrón rítmico diario de la actividad locomotora de los peces se sincroniza a través del ciclo de luz-oscuridad (LO) (Thorpe, 1978). En condiciones constantes de laboratorio, diversos ritmos biológicos continúan expresándose durante días, meses o años, dependiendo de la especie y de las condiciones experimentales. En estos casos, se los denomina ritmos en libre curso y son la expresión de relojes biológicos endógenos. Para examinar el carácter endógeno de los ritmos, se suele aislar a los animales de cualquier estímulo temporal externo mediante el uso de oscuridad constante (OO), luz constante (LL) o pulsos LO ultradianos (Ericsson y Van Veen, 1980). El uso de pulsos LO ultradianos se debe a que, en estudios anteriores con peces, este ciclo
LO ha demostrado ser un factor estabilizador que impide la disociación rápida de múltiples osciladores circadianos (Ericsson y Van Veen, 1980; Sánchez-Vázquez et al., 1996). Aunque el periodo endógeno (tau) que muestran los vertebrados superiores bajo condiciones de curso libre es muy estable, los peces poseen un sistema circadiano flexible, pudiendo mostrar dualidad de fase (dualismo nocturno/diurno) e inestabilidad de tau (Sánchez-Vázquez et al., 1995). Como la duración del periodo de los ritmos circadianos de curso libre suele ser diferente de 24 horas, los organismos ajustan sus marcapasos internos en función de estímulos temporales externos, tales como la disponibilidad de comida, la luz o los cambios diarios de temperatura; aunque la luz es el sincronizador ("zeitgeber") de mayor importancia en la mayoría de los casos (Ali, 1992). Conocer en profundidad los ritmos de las diferentes especies de peces puede ser de utilidad para mejorar la gestión y la práctica de la acuicultura. No obstante, la información disponible sobre los ritmos locomotores de los peces es reducida, y todavía existe cuestionamiento acerca de si las distintas especies muestran ritmos diurnos, nocturnos, crepusculares, o patrones mixtos. Es más, en muchos casos no existe certeza de si los ritmos diarios son inducidos externamente por el ciclo LO o por un marcapasos circadiano interno (Iigo y Tabata, 1996). 